\title{
A case report of serious brain and lung injury caused by mistakenly taking Mosquito repellent liquid contains Meperfluthrin
}

\section{Qing Lou}

Xiamen Children's Hospital

\section{Shengxin Zhang}

Xiamen Children's Hospital

\section{Shengkun Zheng}

Xiamen Children's Hospital

Huaying Liu

Xiamen Children's Hospital

Yi Lu ( $\nabla$ papamaker@126.com )

Xiamen Children's Hospital

\section{Shaoxian Hong}

Xiamen Children's Hospital

\section{Case report}

Keywords: Mosquito repellent, Meperfluthrin, Brain damage, Lung damage

Posted Date: June 24th, 2020

DOI: https://doi.org/10.21203/rs.3.rs-36615/v1

License: (c) (i) This work is licensed under a Creative Commons Attribution 4.0 International License. Read Full License 


\section{Abstract \\ Background}

Mosquito repellent liquid containing Meperfluthrin is widely used in the daily life. Among all clinical cases that children take mosquito repellent by mistake, only a fewwere recorded with serious brain and lung damages caused by Meperfluthrin.

\section{Case presentation:}

This case report presents a 16-month-old child in Xiamen City of China with severe brain and lung injury caused by mistakenly taking mosquito repellent that contains $0.8 \%$ Meperfluthrin. The child was treated with mechanical ventilation, fiberoptic bronchoalveolar lavage, anti-infection, $20 \%$ mannitol for dehydration, ganglioside and neurologic rehabilitation. The child recovered after 2 months.

\section{Conclusion}

This case shows that the improper administration of Meperfluthrin may cause serious toxic damage to brain and lung. Clinicians and parents should pay attention to prevention and targeting treatment promptly after intoxication.

\section{Background}

Meperfluthrin is a pyrethroid insecticide invented and used in China. It is widely used in mosquitorepellent incense and its derivative products because of its high efficiency, low toxicity and convenient use. Among all clinical cases that children taking mosquito repellent by mistakes, only a few were recorded serious poisoning symptoms caused by Meperfluthrin. We have only retrieved one serious poisoning case caused by mistakenly taking mosquito repellent liquid containing Meperfluthrin reported by Li Jiru, a doctor from Shengjing Hospital affiliated to Shenyang Medical University of China in July 2019[2], and now we report a case of persistent convulsion, progressive lung damage and brain damage caused by the accidental administration ofmosquito repellent liquid containsMeperfluthrin.

\section{Case Presentation}

On June 28, 2019, a 16-month-year old male with coma and convulsion was admitted to the ER of Xiamen Children's Hospital. No history of food poisoning was reported at that time, and there was no medical history before the admission. The physical examination showed: T $35.6{ }^{\circ} \mathrm{C}, \mathrm{P} 161 \mathrm{times} / \mathrm{min}, \mathrm{BP}$ $75 / 35 \mathrm{mmHg}(1 \mathrm{mmHg}=0.133 \mathrm{kPa}), \mathrm{R} 58$ times/min, oxygen saturation was $56 \%$ without oxygen inhalation. Patient was unconscious with short of breath, convulsed, and both eyes quaked horizontally. Patient has cyanosis, with $3 \mathrm{~mm}$ diameter in pupils, showed slow light reflex, neck resistance was normal, 
flapping of nasal alae, positive triple-concave sign, coarse respiratory sounds in both lungs, and a few wet rales, CRT > $2 \mathrm{~s}$. Chloral hydrate, phenobarbital, midazolam and propofol were given in order for sedation, the convulsion lasted for about 35 minutes and relieved after treatment, dyspnea was observed after sedation. Patient was treated with mechanical ventilation, and propofol was then used to help the patient to maintain sedated for chest radiography (Figure 1A: shows a little exudation of the lungs) and craniocerebral CT (Figure 2: no obvious abnormalities on craniocerebral CT). Patient was then transferred to PICU. Fibrobronchoscopy was conducted on the day of admission. Result showed hyperemia and edema on mucous membrane without erosion and foreign body (Fig. 3A, B). Results of blood test were as follows: WBC 23.6x109/; CRP $116.4 \mathrm{mg} / \mathrm{L}$; PCT $6.67 \mathrm{ng} /$. The result of arterial blood gas analysis showed: $\mathrm{pH}=7.218, \mathrm{PCO} 248.1 \mathrm{mmHg}, \mathrm{PO} 276 \mathrm{mmHg}, \mathrm{HCO} 3-19.6 \mathrm{mmol} / \mathrm{L}, \mathrm{BE}-14 \mathrm{mmol} / \mathrm{L}, \mathrm{Lac} 4.280$ $\mathrm{mmol} / \mathrm{L}$, AG $5 \mathrm{mmol} / \mathrm{L}$.

The patient developed a fever of $38.5^{\circ} \mathrm{C}$ on the second day of admission, with increased lung rales, short of breath and dyspnea. Multiple patchy shadows in both lungs were seen on the bedside chest radiograph (Fig. 1B), which was more progressive than the day of admission. Methylprednisolone was prescribed by the patient's weight $(1 \mathrm{mg} / \mathrm{kg})$ and used twice a day to resist inflammation; budesonide for atomization, and injection of Mucosolvan was applied for the purpose of resolving phlegm. Bedside chest radiograph was performed on the third (Fig.1C) and fifth day (Fig.1D) after admission, respectively. Fig.1C showed the continued exudation in both lungs and atelectasis appeared; Fig.1D showed atelectasis in the right lower lobe and the left upper lobe, and multiple strips of dense shadows in both lungs. On the 6th day after admission, more secretions were showed in bronchofibroscopy (Fig. 3C), and alveolar lavage was used. The temperature dropped to normal on the 7 th day after admission.

The patient was withdrawn from the ventilator on the 15th day after admission. Oxygen saturation was back to normal under nasal catheter oxygen inhalation. Blood test result and CRP were normal. After discontinuation of sedative treatment, patient regained consciousness but with sluggish reaction. Patient was unable to speak and laugh, and there is paroxysmal limb involuntary shaking, slightly increased muscle tension. EEG showed the diffused 2.0-4.0 hz slow wave activity (Fig.5). Cranial MRI showed abnormal signal beside the anterior horn of the right lateral ventricle (Fig.6), therefore softening focus was considered. Meperfluthrin poisoning was considered by the information that "an empty insect repellent bottle was found beside the patient in the incident scene" provided by patients' parents at that time. Therefore, ganglioside nutrition brain cells and hyperbaric oxygen treatment were prescribed. The bedside X-ray chest film (Fig. 1E) showed significant absorption of lung exudation on the 20 days after admission, and patient exhibited an improved reaction, with limb tremor eased, patient was then transferred to the rehabilitation center. The patient was fully recovered in the follow-up session after two months of discharged.

\section{Discussion And Conclusions}

Pyrethroid pesticides are one of the most widely used agricultural and household pesticides, accounting for nearly $25 \%$ of the global pesticide market [3]. Poisoning incidents caused by pyrethroid pesticides are 
frequently reported. According to the latest statistic of the Toxicant Exposure Monitoring System of the American Association of Poison Control Centers, pesticide poisoning ranks the ninth among all types of poisoning, which accounts for $3.28 \%$ of all intoxication incidents. In China, chenshuyang et Al. [5] analyzed and found 2954 reported cases of pesticide poisoning from 1992 to 2003 , accounting for $3.21 \%$ of all poisoning cases. Meperfluthrin is also a kind of pyrethroid insecticide, which is often used for mosquito incense, mosquito incense tablets and liquid mosquito incense products, with a concentration of $0.8 \%$, few with concentration of $0.6 \%$, and widely used in Chinese families.

Pyrethroid pesticides are classified into two types based on different chemical structure. Type I does not contain the cyano group (such as pyrethrin) but type II does (such as cypermethrin, deltamethrin) ${ }^{[6]}$, which is alipid-soluble chemical compound that can pass through the blood-brain barrier effectively. It mainly targets and changes the sensitivity of the voltage-gated sodium channels, which keeps the sodium channels open, so the sodium ions flow into the cell and prolong the depolarization, and causes abnormal excitation of the nerve cells. It has been shown that sodium channels in the dorsal root ganglion and peripheral nerves of mammals are more sensitive to type II pyrethroids than type I before sexually mature, and type II can also keep the sodium channel open longer than type I. It has also been suggested that type II pyrethroids can control the voltage-dependent chloride channels in the brain, nerves, muscles and salivary glands, thereby reducing the chloride channel currents ${ }^{[7]}$. At higher concentrations, pyrethroids can act on the GABA-gated chloride channels, leading to severe type II toxic convulsions. Romero et al ${ }^{[8]}$ found that pyrethroid metabolism produces superoxide anion, hydroxyl radicals and hydrogen peroxide free radicals. Kanbur et al ${ }^{[9]}$ found that cypermethrin exposure increases oxidative stress (malondialdehyde, nitric oxide) in the rat brain, while superoxide dismutase(SOD), antioxidant enzymes such as catalase(CAT) and glutathione peroxidase(GSH-Px) decreased significantly, and this phenomenon became more obvious with prolongation of exposure time.

Because sodium channels are located in type I and type II alveolar epithelium, the permeation gradient may be destroyed by pyrethroids, leading to airway and bronchial mucosal edema ${ }^{[10]}$. Due to the hypersensitivity caused by pyrethroids, the ongoing inflammatory reaction may also be one of the factors that cause lung damage. In addition, the damages caused by certain organic solvents in pyrethroid drugs, such as chloroform, benzene and ether, may also be the cause of lung lesions.

Pyrethroid drugs are often taken by mistake, mild poisoning can be manifested as upper respiratory tract irritation, cough, sore throat, dizziness, headache, nausea, vomiting, abdominal pain, fatigue, chest tightness, sensory abnormalities. Serious poisoning may cause disturbance of consciousness, seizures, severe pneumonia, dyspnea, cyanosis and coma ${ }^{[6]}$, death is more unlikely to occur ${ }^{[11]}$. Neurological symptoms are one of the main clinical manifestations ${ }^{[12]}$, type II poisoning can cause muscle clonus and paroxysmal tonic convulsion, similar to epilepsy ${ }^{[13]}$. The concentration of the drug in this case is $0.8 \%$, and it caused severe convulsion, shortness of breath, dyspnea and progressive lung injury and brain damage. The patient recovered fully after treatment. 
When patient is diagnosed with pyrethroid poisoning, $2 \%-4 \%$ sodium bicarbonate should be used for gastric lavage, and then medicinal carbon should be applied for absorbing the residual solution in the stomach. Because the toxin can cause serious lung injury and nervous system damage, active measures such as controlling static shock, reducing cranial pressure, respiratory support; mechanical ventilation, corticosteroids injection, anti-oxidative stress, infection prevention should be considered in sever case. Atropine in small dosage can also be applied, but atropinization is not recommended. Although pyrethroids belong to the low toxic drugs, they are more likely to cause continuous convulsion, progressive lung injury and even ARDS. Therefore, blood purification should be carried out as early as possible.

Because pyrethroid insecticides are widely used, medical staff needs to pay more attention on health education, reminding parents to prevent children from taking them by mistake. Meanwhile, manufacturers also need tobe alter and have the emergency directions for mistakenly intake, so as to improve the awareness of poisoning and minimize such accidental injuries.

\section{Declarations}

Ethics approval and consent to participate

The case report has been approved by the Ethics Management Committee of Xiamen Children's Hospital

Consent for publication

Not applicable

Availability of data and materials

Not applicable

Authors' contributions

All authors read and approved the final manuscript.

Funding

Not applicable

Competing interests

The authors declare that they have no competing interests.

Acknowledgements

Not applicable 


\section{References}

1. QiMingzhu Z, Jingmei, et al.development and application of Meperfluthrin [J].chinese medical insecticide, 2010,16 (3): 172-174.

2. LiJiru W, Lijie, Guan WH, et al.a case of oral flufluthrin poisoning and literature review [J].chinese pediatric emergency medicine, 2019,26 (7): 558-560.

3. Shafer TJ, Meyer DA, Crofton KM. Developmental neurotoxicity of pyrethroid insecticides: critical review and future research needs [J]. Environmental health perspectives. 2005;113(2):123-36.

4. Gummin DD, Mowry JB, Spyker DA, et al. 2017 Annual Report of the American Association of Poison Control Centers' National Poison Data System (NPDS): 35th Annual Report [J]. Clinical toxicology (Philadelphia, Pa), 2018, 56(12): 1213 - 415.

5. Chenshuyang W, Hongfei. Yin Yu.epidemiological characteristics of pesticide poisoning and status of pesticide poisoning reports in China [J].china Journal of Labor Health and Occupational Diseases, 2005,05 (in Chinese): 336-9.

6. Bradberry SM, Cage SA, Proudfoot AT, et al. Poisoning due to pyrethroids [J]. Toxicological reviews. 2005;24(2):93-106.

7. ForshawPJ,Ray. DE.A novel action of deltamethrin on membrane resistance in mammalian skeletal muscle and non-myelinated nerve fibres[J].Neuropharmacology,1990,29(1):75-81.

8. Romer OA, Ramos E, Castellano V, et al. Cytotoxicity induced by deltamethrin and its metabolites in SH-SY5Y cells can be differentially prevented by selected antioxidants [J]. Toxicology in vitro: an international journal published in association with BIBRA, 2012, 26(6): 823-30.

9. Kanbur M, Silig Y, Eraslan G, et al. The toxic effect of cyMeperfluthrin, amitraz and combinations of cyMeperfluthrin-amitraz in rats [J]. Environ Sci Pollut Res Int. 2016;23(6):5232-42.

10. GognaA.Hypersensitivity reaction. and acute respiratory distress syndrome in pyrethriod poisoning and role of steroid therapy[J].Asia Pacific J Med Toxicol,2015(4):91-93.DOI:10. 22038/APJMT.2015.3732.

11. Cha YS, Kim H, Cho NH. etal.Pyrethriod poising: features and predictors of atypical presentations[J]. Emerg Med J. 2014;31(11):899-903.

12. He F, Wang $\mathrm{S}$, Liu L, et al. Clinical manifestations and diagnosis of acute pyrethroid poisoning [J]. Arch Toxicol. 1989;63(1):54-8.

13. Yang Jian C, Gaohong P, Ying, et al.diagnosis and treatment of pyrethroid pesticide poisoning [J].laboratory medicine and clinic, 2011,8 (16): 2000-2001.

14. Walters JK, Boswell LE, Green MK, et al.Pyrethrin and prethroid illnesses in the Pacific northwest: a five-year review[J].Public Health Rep,2009,124(1):149-159.

\section{Figures}




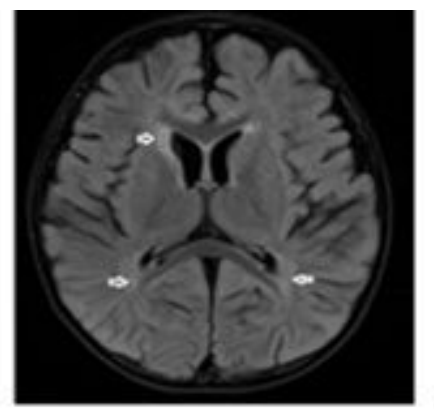

Fig.6 Cranial MRI

Cranial MRI showed abnormal signal beside the anterior horn of the right lateral ventricle

\section{Figure 1}

\section{Fig6}

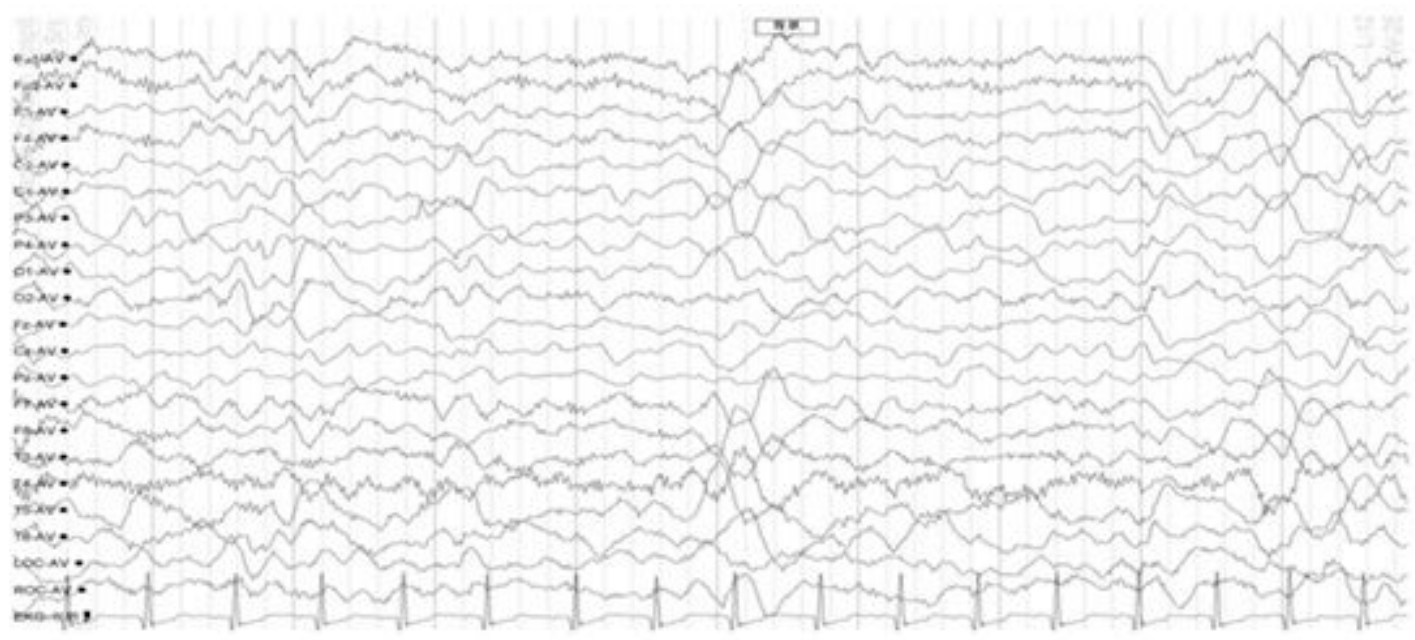

Fig.5 EEG shows the diffused $2.0-4.0$ hz slow wave activity

\section{Figure 2}

\section{Fig5}
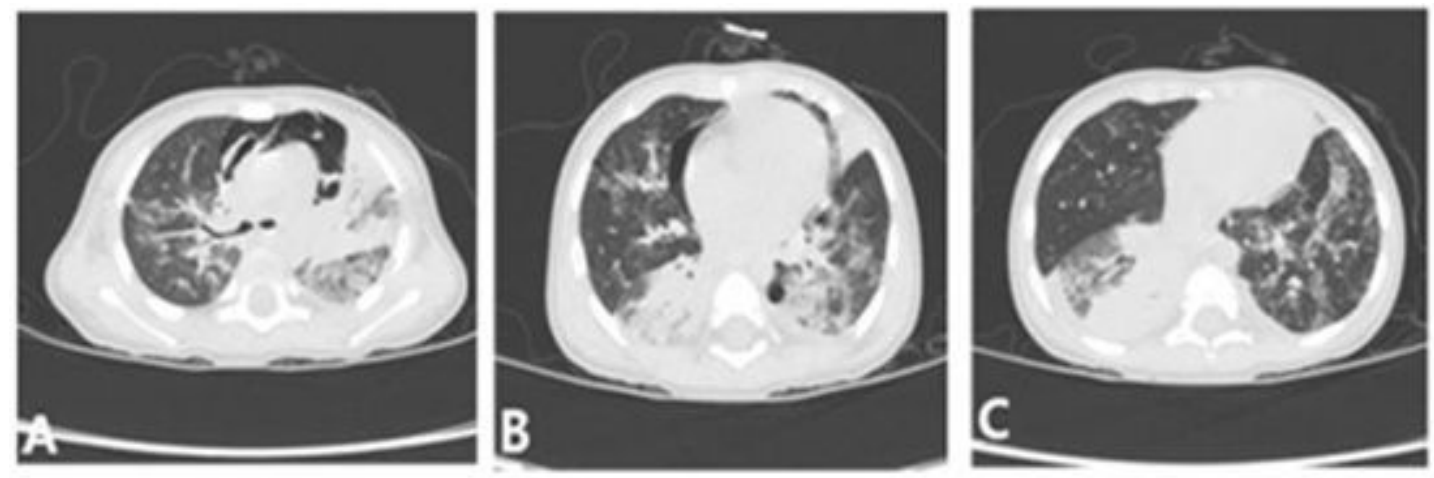

Pic.4 Lung CT image

Both lungs show multiple striated exudation, atelectasis of right lower lobe and left upper lobe

\section{Figure 3}



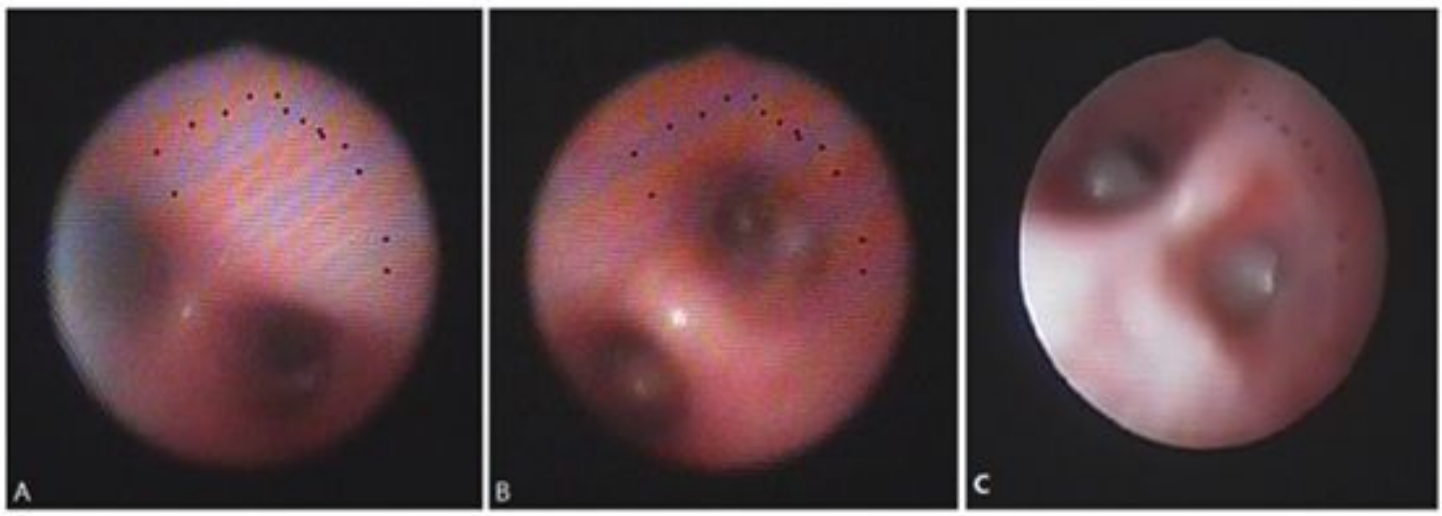

Fig. 3 Bronchogram

A. B. shows congestion of bronchial mucosa under fiberoptic bronchoscopy on the day of admission

C. Trachea secretion under fiberoptic bronchoscopy on the $6^{\text {th }}$ day after admission

\section{Figure 4}

Fig3
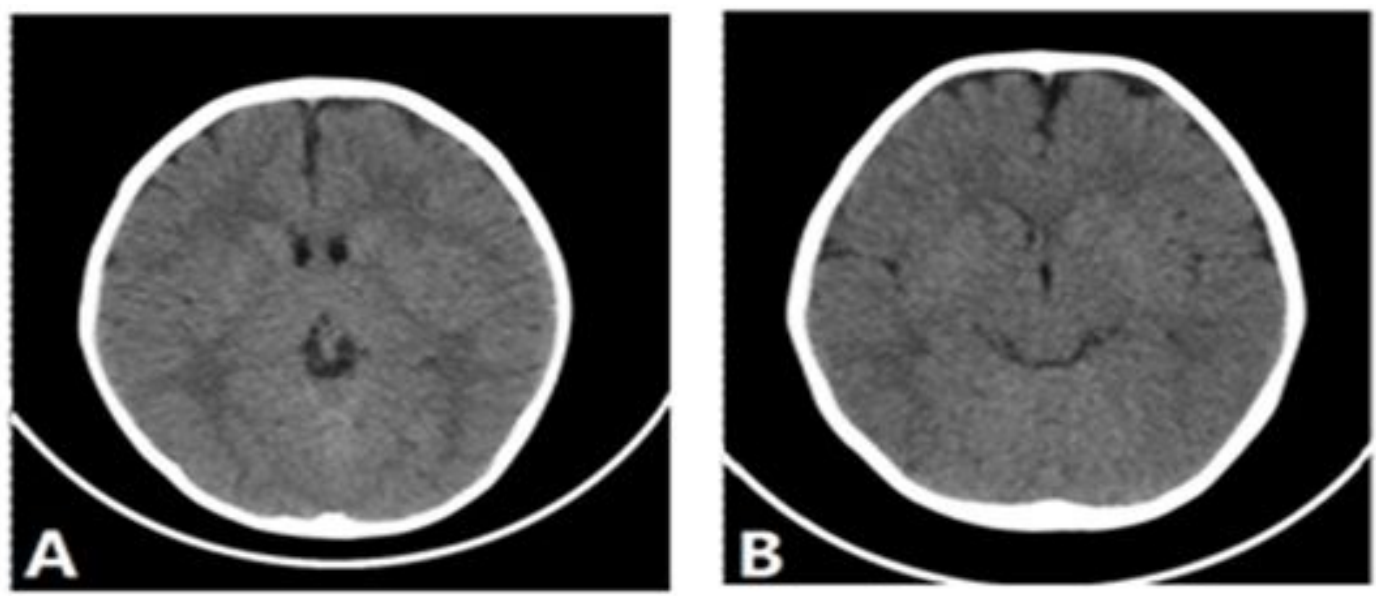

Fig. 2 Brain CT scan

A. B. The brain CT scan result shows no abnormality

Figure 5

Fig2 

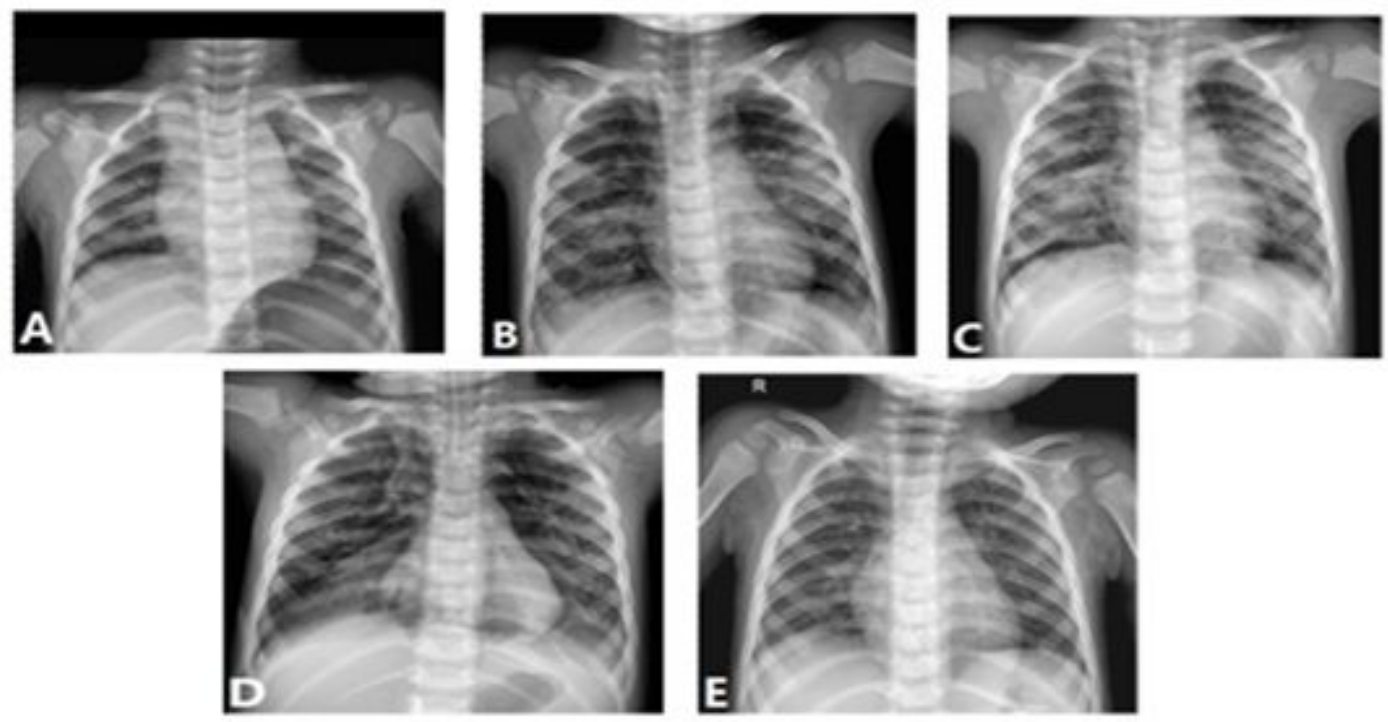

Fig. $1 X$-ray image of Lung

A. A few exudations were seen after intubation on the day of admission B.C. Pathological changes in both lung on the $2^{\text {nd }}$ and $3^{\text {rd }}$ day after admission D. Lung atelectasis was showed on the fifth day of admission E. On the 20th day of admission, showed exudation were absorbed in both lungs

\section{Figure 6}

Fig1 